Nervenarzt 2014 $\cdot 85: 1231-1232$

DOI 10.1007/s00115-014-4045-3

Online publiziert: 17. September 2014

๑) Springer-Verlag Berlin Heidelberg 2014

W. Maier ${ }^{1} \cdot$ W. Oertel ${ }^{2}$

${ }^{1}$ Klinik und Poliklinik für Psychiatrie und Psychotherapie, Universitätsklinikum Bonn

${ }^{2}$ Klinik für Neurologie, Philipps-Universität Marburg

\title{
Prädiktion neurologischer und psychiatrischer Erkrankungen
}

men, sodass Ärzte von Patienten und Ratsuchenden vermehrt nach künftigen Krankheitsrisiken und deren Verhütung gefragt werden. Diese Entwicklung wird auch per Internet und Druckmedien mit regelmäßig eintreffenden Meldungen gefördert, wer und in welchem Maß für eine neuropsychiatrische Erkrankung gefährdet ist und wie man seinen eigenen Risikoscore für z. B. Alzheimer-Demenz, Depression, Psychose oder Schlaganfall anhand simpler Fragebögen per Mobil-Phone berechnen kann.

- Drittens ist der Arzt - im Sinne des medizinethischen Prinzips der Benefizienz - zur Aufklärung über künftige Gesundheitsrisiken verpflichtet. Entsprechend stellt auch das Sozialgesetzbuch fest: Prävention vor Therapie.

- Viertens wurde auf nationaler und europäischer Ebene erkannt, welche immensen Kosten (derzeit jeder dritte Euro in den Gesundheitssystemen Europas; Stichwort DemographieGipfel; www.gesundheitsziele.de) für die Diagnose, Therapie und Pflege von ZNS-Krankheiten auf die Gesellschaft zukommen.

- Fünftens haben Grundlagenforschung und klinische Neurowissenschaften in den letzten Jahren klargestellt, dass den Symptomen vieler Gehirnerkrankungen (z. B. Alzheimer, Parkinson) jahre- bis jahrzehntelange neurobiologische Krankheitsprozesse vorausgehen. Diese neurologischen, präklinischen Entwicklungen können zunehmend durch Biomar- ker und Neuroimaging bei Gesunden dargestellt werden. Hier eröffnet sich ein zeitliches Fenster für gezielte Prävention und Frühintervention.

- Sechstens sind damit Politik, forschungsfördernde Institutionen, Krankenkassen und die forschende pharmazeutische Industrie zu einem Umdenken gezwungen: Nicht mehr die Entwicklung weiterer (noch besserer) symptomatischer Behandlungsformen ist gefragt, sondern das primäre Ziel der Therapieforschung und medizinischen Versorgung muss die Prävention (das Verhindern der Manifestation von Erkrankungen) sein. Und diese macht nur Sinn, wenn anhand gesicherter Erkenntnisse das Auftreten von Krankheiten in (Subgruppen) der Bevölkerung mit hoher Wahrscheinlichkeit vorausgesagt werden kann.

Daher das Thema „Prädiktion“.

Mit diesem Leitthemenheft wollen wir uns der Thematik stellen. Sechs Experten(-gruppen) haben sich der Mühe unterzogen, für sechs wichtige Erkrankungen der Neurologie und Psychiatrie [Alzheimer-Demenz (Jessen, Dodel), Psychosen (Klosterkötter), Depression (Himmerich und Kollegen), Multiple Sklerose (Wiendl, Tackenberg), Angststörungen (Domschke), Schlaganfall (Hamann)] den derzeitigen Stand der Prädiktionsforschung kritisch zu würdigen. Hier wird beeindruckend dargestellt, wie viel wir bereits über Voraussagen der referierten Erkrankungen aufgrund der Studienergeb- 
nisse aus großen Kollektiven wissen. Andererseits wird am Beispiel des „offenen Foramen ovale“ im Beitrag „Schlaganfall - Hamann" dargelegt, wie Prädiktion manipuliert werden kann. Und die Beiträge verdeutlichen das grundsätzliche Dilemma der Prädiktion, wenn es darum geht, sie auf das individuelle Niveau des einzelnen Patienten herunterzubrechen. Prädiktion im Einzelfall erfordert nämlich hohe Präzision und genaue Spezifität; dieses Prinzip gilt insbesondere dann, wenn es um eine nebenwirkungsträchtige Präventionsmaßnahme geht. Hier sind die ärztliche Kunst in der individuellen Einschätzung und das persönliche Gespräch zwischen Arzt und Patient mehr denn je gefordert, um kollektive Vorhersagen auf die individuelle Situation des einzelnen Patienten zu transferieren.

Das vorliegende Heft ist ein erster Schritt. Insgesamt ist die Übersicht ermutigend: Die jetzt bereits vorhandenen Ansätze lassen erkennen, dass es möglich ist, erste Präventionsstudien z. B. im prodromalen Stadium der Alzheimer-Demenz zu planen und umzusetzen oder mit relativ simplen Mitteln das Risiko des Auftretens eines ersten oder erneuten Schlaganfalls zu reduzieren. Wir hoffen, dass Ihnen das vorliegende Heft eine nützliche Grundlage für Ihre berufliche Tätigkeit in Fragen der Prädiktion und der sich hieraus ableitenden Prävention wichtiger neurologischer und psychiatrischer Erkrankungen liefert und dazu beiträgt, Ihre Patienten gut zu beraten.

Mit freundlichen kollegialen Grüßen

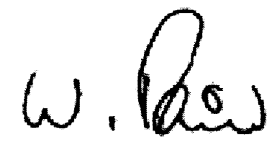

Wolfgang Maier

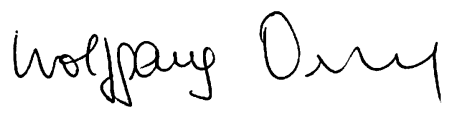

Wolfgang Oertel

\section{Korrespondenzadressen}

Prof. Dr. W. Maier

Klinik und Poliklinik für Psychiatrie und

Psychotherapie, Universitätsklinikum Bonn

Sigmund-Freud-Str. 25, 53105 Bonn

Wolfgang.Maier@ukb.uni-bonn.de

Prof. Dr. Dr. h.c. W. Oertel

Klinik für Neurologie,

Philipps-Universität Marburg

Baldingerstraße, 35043 Marburg

oertelw@med.uni-marburg.de

Interessenkonflikt. W. Maier und W. Oertel geben an, dass kein Interessenkonflikt besteht.

\section{Robert-Wartenberg-Preis 2015}

Die leitenden Klinikneurologen Deutschlands schreiben für 2015 die Robert-

Wartenberg-Lecture aus. Der Preis ist mit einer Vorlesung auf der Jahrestagung der Leitenden Klinikneurologen in Kassel am 27.02.2015 verbunden und wird mit 5.000 Euro dotiert.

Der Preis richtet sich an wissenschaftlich, jedoch nicht an universitären Einrichtungen tätige Neurologen (primär Oberärzte, Fachärzte, Assistenzärzte), deren Arbeiten Bezug zur klinischen Neurologie haben und konkret Anwendung finden können. Es soll eine bereits veröffentlichte, eine zur Veröffentlichung angenommene wissenschaftliche Einzelarbeit oder ein für den Bewerber repräsentatives wissenschaftliches Werk (an einer nicht-universitären Einrichtung entstanden oder dort fortgesetzt) zusammen mit einem Curriculum vitae und einem Schriftenverzeichnis eingereicht werden. Einsendungen bitte bis spätestens 15 .

Dezember 2014 in schriftlicher Form und zusätzlich in elektronischer Form (PDF-

Datei) an:

Prof. Dr. Karl Wessel Neurologische Klinik, Klinikum Braunschweig

Salzdahlumer Straße 90

38126 Braunschweig

Tel: 0531/5952300

k.wessel@klinikum-braunschweig.de

Quelle: Neurologische Klinik des Städtischen Klinikums Braunschweig dGmbH 